\title{
Endoscopic Transversal Incision and Longitudinal Septostomy (TILS): An Updated Technique for Treating Esophageal Diverticulum
}

\author{
$\mathrm{Hao} \mathrm{Hu}^{\mathrm{a}}$ Min Wang ${ }^{\mathrm{b}}$ Liang Zhu ${ }^{\mathrm{a}}$ Pinghong Zhou ${ }^{\mathrm{a}}$ \\ aEndoscopy Center and Endoscopy Research Institute, Zhongshan Hospital, Fudan University, Shanghai, China; \\ ${ }^{b}$ Department of Gastroenterology, Hepatology and Nutrition, Shanghai Children's Hospital, Shanghai Jiaotong \\ University, Shanghai, China
}

\section{Keywords}

Endoscopic treatment - Esophagus - Diverticular disease

\begin{abstract}
An esophageal diverticulum is a protruding pouch in a weak portion of the esophageal lining. Previously, our team had reported an endoscopic tunneling technique (submucosal tunneling endoscopic septum division) for diverticulum treatment. However, it does not perform well for the diverticulum located in the upper esophagus, where most diverticula are located. Herein, we report a new endoscopic technique, called endoscopic transversal incision and longitudinal septostomy (TILS). TILS provides both larger operational spaces and complete septostomy and can be performed on most types of diverticula, including Zenker's diverticulum.

(c) 2020 S. Karger AG, Basel
\end{abstract}

\section{Introduction}

An esophageal diverticulum is a protruding pouch in a weak portion of the esophageal lining. Esophageal diverticulum can affect individuals of all ages; however, it tends to manifest in elderly and debilitated patients [1]. Thus, its treatment needs to be less invasive with fewer complications. Previously, our team had reported an endoscopic tunneling technique (submucosal tunneling endoscopic septum division [STESD]) for diverticulum treatment [2]. STESD exploits the tunneling technique to achieve complete septum dissection, which helps maintain mucosal integrity. But in our daily clinical practices, STESD sometimes does not perform well for the diverticulum located in the upper esophagus, where making a submucosal tunnel is not easy. Considering the most common type of esophageal diverticulum, Zenker's diverticulum (ZD), usually locates in the upper part of the esophagus, an updated endoscopic treatment is necessary. Herein, we report a new endoscopic technique called endoscopic transversal incision and longitudinal septostomy (TILS). As the technique does not involve a submucosal tunnel, TILS can be performed on most types of diverticula. Meanwhile, transversal incision provides TILS a larger operational space and complete septostomy (Fig. 1).

\section{Description of the Technology}

The patient is kept under general anesthesia with endotracheal intubation. Prophylactic antibiotics are given intravenously $30 \mathrm{~min}$ before the procedure. Carbon di-

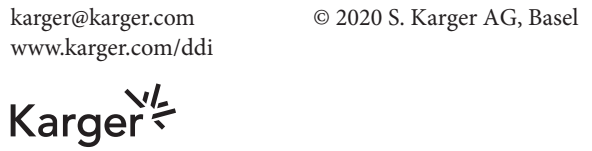


Fig. 1. Illustration of TILS technique. a ZD (black arrow). b Transverse incision made on the diverticular septum, clearly exposing the muscle fibers. c Septum division dissected in the middle. d Closure of the mucosal wound with clips. TILS, transversal incision and longitudinal septostomy; ZD, Zenker's diverticulum.
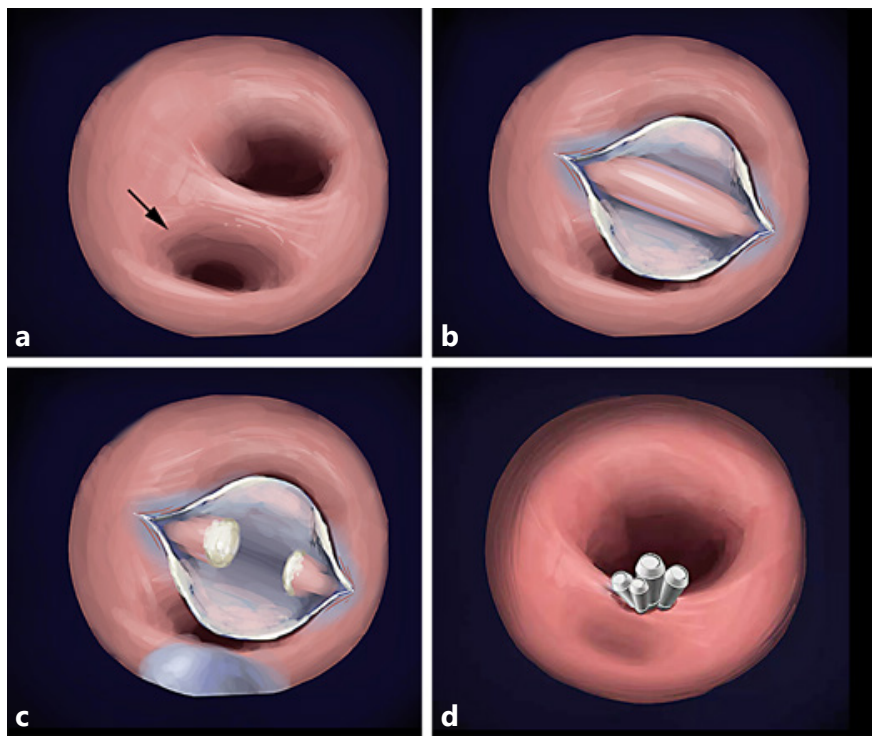
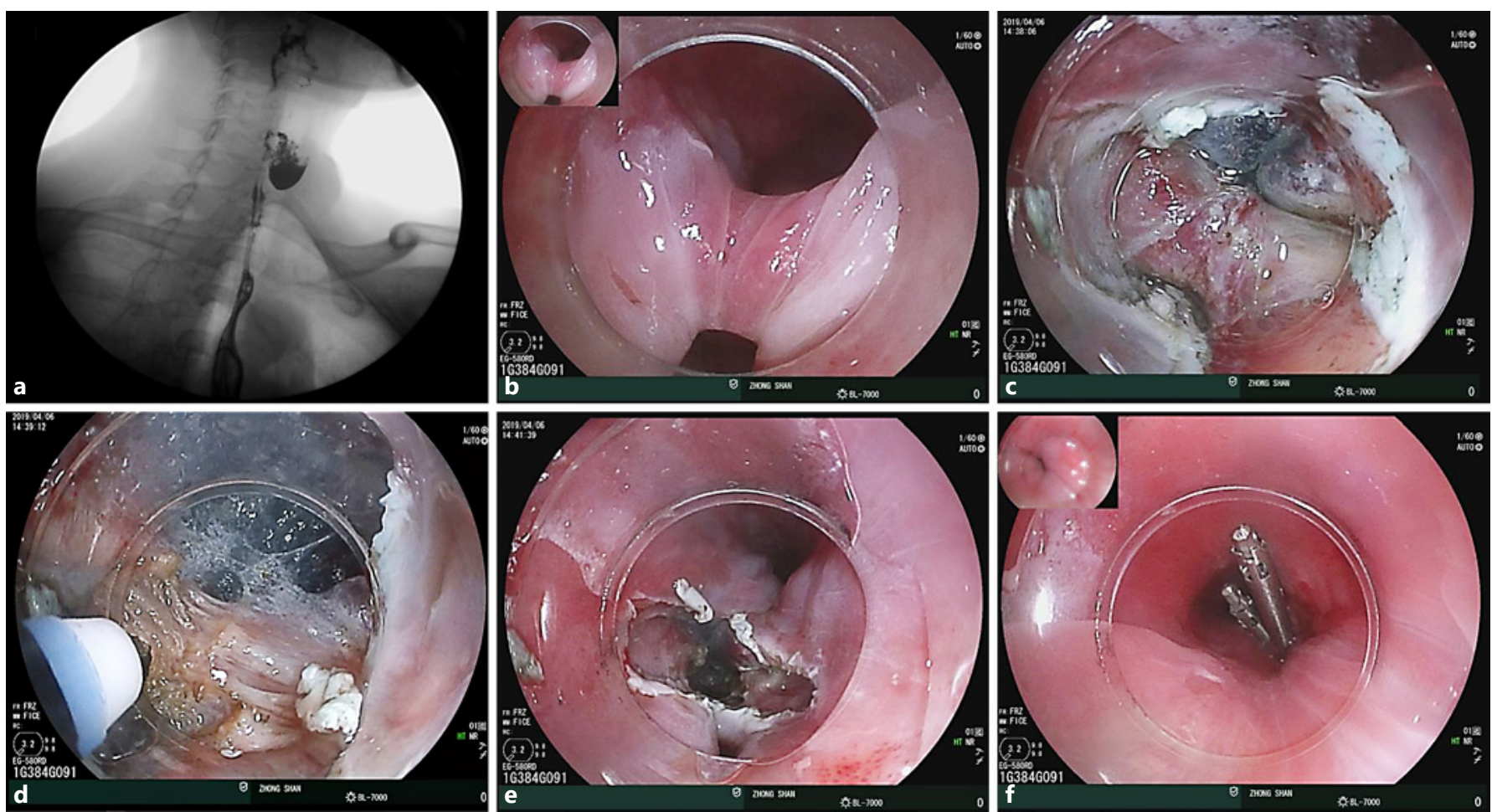

Fig. 2. TILS for the treatment of ZD in the upper esophagus. a Visualization of the diverticulum under barium swallow (arrow). b A $20-\mathrm{mm} \mathrm{ZD}$ located $15 \mathrm{~cm}$ from the incisors (arrow). c Transverse incision made on the mucosa of the diverticular septum, clearly exposing the muscle fibers. $\mathbf{d}$ The septum dissected in the middle and protective water space around can be seen. e Completion of myotomy. $\mathbf{f}$ Mucosal closure. TILS, transversal incision and longitudinal septostomy; ZD, Zenker's diverticulum. 


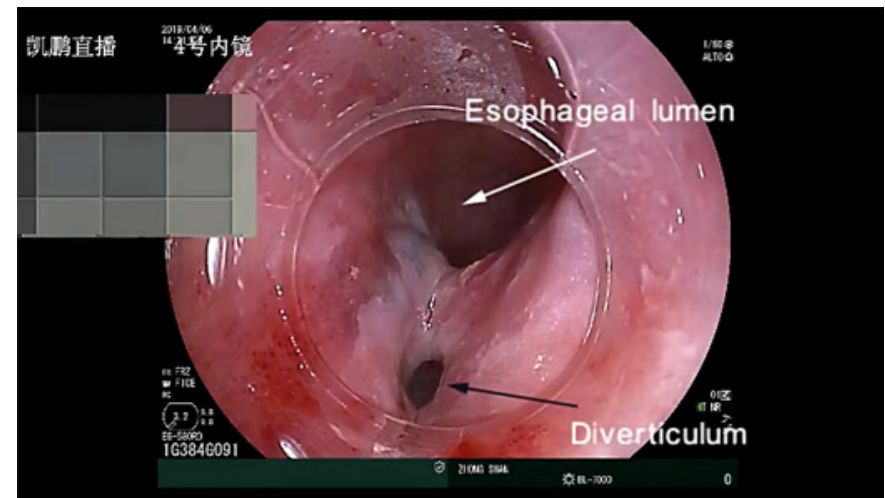

Video 1. TILS procedure performed on a 50-year-old female patient.

oxide is routinely used for gas insufflation. The TILS procedure consists of 3 steps. Step 1 is performing a transverse incision on the septum. The transverse incision along the long axis of the septum is made with a HybridKnife (ERBE; Erbe Elektromedizin GmbH, Tübingen, Germany) to expose the cricopharyngeal muscle fibers. A VIO electrosurgical generator (VIO 200D) was used, and Endo-cut Q mode (effect 3, duration 2, interval 4) with forced coagulation (effect 2, $50 \mathrm{~W}$ ) was employed. Step 2 is performing septostomy. To safely isolate cricopharyngeal muscle fibers, a protective submucosal water space is carefully established by using the HybridKnife, and water injection and submucosal cutting manipulations alternate in this step. Then the septum is dissected in the middle, ending 1-2 cm distal to the bottom of the diverticulum. Step 3 is mucosal closure. After careful hemostasis, the mucosal incision is sealed with metal clips (Fig. 2).

\section{Video Description}

Video 1 shows the TILS procedure on a 50-yearold female patient. This patient had complaints of dysphagia and halitosis for 5 years. Gastroscopy and $\mathrm{X}$-ray examination revealed a 20 - $\mathrm{mm}$-diameter ZD located $15 \mathrm{~cm}$ from the incisors. The $\mathrm{ZD}$ was successfully treated by TILS in 15 min with no obvious adverse effects. The patient was administered clear fluid on postoperative day 1 and discharged on day 2. The symptoms of dysphagia and bad breath disappeared at the 1-month follow-up.

\section{Discussion}

The main advantage of TILS is that it does not require a submucosal tunnel. TILS can be easily applied to the diverticulum in the upper esophagus where STESD can be hardly performed because STESD usually requires a 1.5 - to $2.0-\mathrm{cm}$ longitudinal incision for endoscope entry and an approximately $3-\mathrm{cm}$ length submucosal tunnel [2]. Furthermore, the technical difficulty is greatly reduced as even experienced endoscopists without expertise of submucosal tunneling can perform TILS. Another advantage of TILS is making a transverse incision on the diverticulum septum. This is the major difference from other current endoscopic methods, in which the mucosa and septum muscle are cut longitudinally in the middle $[3,4]$. Transverse incision provides TILS a larger operational space and better surgical view so that a complete and accurate septostomy can be precisely achieved. This is important for the successful treatment of a diverticulum, as incomplete dissection leads to residual diverticulum and recurrence [5]. In the meantime, enough operational space likely reduces common complications, such as mucosal injury or perforation, leading theoretically to a lower risk of postoperative leakage and mediastinitis. It should be noted that other endo-knives, such as hook knife and triangle tip knife, can also be used for this technique. To date, TILS has been successfully performed in our center with no severe complications.

In conclusion, TILS is a new technique for treating esophageal diverticulum, especially the diverticulum in the upper portion of the esophagus. Further clinical studies with more patients and multicenter data regarding efficacy and safety are nonetheless needed.

\section{Statement of Ethics}

Written informed consent was obtained from the patient, and the study protocol was approved by the Ethics Committee of Zhongshan Hospital.

\section{Disclosure Statement}

The authors have no conflicts of interest to declare. 


\section{Funding Sources}

This study was supported by a municipal human resources development program for outstanding young talents in medical and health sciences in Shanghai (2018YQ33), and grants from the Natural Science Foundation of Shanghai (18411952500) and the National Natural Science Foundation of China (81701750 and 81900548).

\section{Author Contributions}

Study concept and design: Pinghong Zhou. Acquisition of data: $\mathrm{Hao} \mathrm{Hu}$, Min Wang, and Liang Zhu. Manuscript writing: $\mathrm{Hao} \mathrm{Hu}$ and Min Wang.

\section{References}

1 Beard K, Swanström LL. Zenker's diverticulum: flexible versus rigid repair. J Thorac Dis. 2017;9(Suppl 2):S154-62.

2 Li QL, Chen WF, Zhang XC, Cai MY, Zhang YQ, Hu JW, et al. Submucosal tunneling endoscopic septum division: a novel technique for treating Zenker's diverticulum. Gastroenterology. 2016;151(6):1071-4.
3 Ishaq S, Sultan H, Siau K, Kuwai T, Mulder CJ, Neumann H. New and emerging techniques for endoscopic treatment of Zenker's diverticulum: state-of-the-art review. Dig Endosc. 2018;30(4):449-60.

4 Tang SJ, Lara LF. Flexible endoscopic clip-assisted Zenker's diverticulotomy (with videos). Gastrointest Endosc. 2008;67(4):704-8.
5 Costamagna G, Iacopini F, Bizzotto A, Familiari P, Tringali A, Perri V, et al. Prognostic variables for the clinical success of flexible endoscopic septotomy of Zenker's diverticulum. Gastrointest Endosc. 2016;83(4):76573. 